\title{
THE OUTER SPECTRAL RADIUS AND DYNAMICS OF COMPLETELY POSITIVE MAPS
}

\author{
J. E. PASCOE
}

\begin{abstract}
We examine a special case of an approximation of the joint spectral radius given by Blondel and Nesterov, which we call the outer spectral radius. The outer spectral radius is given by the square root of the ordinary spectral radius of the $n^{2}$ by $n^{2}$ matrix $\sum \overline{X_{i}} \otimes X_{i}$. We give an analogue of the spectral radius formula for the outer spectral radius which can be used to quickly obtain the error bounds in methods based on the work of Blondel and Nesterov. The outer spectral radius is used to analyze the iterates of a completely positive map, including the special case of quantum channels. The average of the iterates of a completely positive map approach to a completely positive map where the Kraus operators span an ideal in the algebra generated by the Kraus operators of the original completely positive map. We also give an elementary treatment of Popescu's theorems on similarity to row contractions in the matrix case, describe connections to the Parrilo-Jadbabaie relaxation, and give a detailed analysis of the maximal spectrum of a completely positive map.
\end{abstract}

\section{Contents}

1. Introduction 2

1.1. Dynamics of quantum channels and other completely positive maps

1.1.1. The general case

1.1.2. Nondegenerate maximal spectrum and quantum channels

1.2. The outer spectral radius formula and the Blondel-

Nesterov approximation

1.3. A Popescu-Rota-Strang theory of the outer spectral radius

2. Preliminaries

2.1. The $\psi$ involution

2.2. The partial trace $Q$

3. The outer spectral radius formula

4. Construction of the Lyapunov matrix

Date: May 27, 2019. 
5. The outer spectrum and dynamics of completely positive maps

5.1. Maximum eigenvalue is nonnegative 17

5.2. Structure of the $T_{\lambda} \quad 18$

5.3. Spectral structure of $\sum X_{i}^{\otimes 2 k} \quad 20$

References

\section{InTRODUCTION}

The spectral radius of a matrix $X \in M_{n}(\mathbb{C})$, denoted $\rho(X)$ is given by the maximum modulus of the eigenvalues of $X$. The spectral radius can also be computed via the Gelfand formula:

$$
\rho(X)=\lim _{k \rightarrow \infty}\left\|X^{k}\right\|^{1 / k} .
$$

The joint spectral radius of a tuple $\left(X_{1}, \ldots, X_{d}\right) \in M_{n}(\mathbb{C})^{d}$ is defined in terms of a Gelfand type formula to be:

$$
\rho\left(X_{1}, \ldots, X_{d}\right)=\lim _{k \rightarrow \infty} \sup _{1 \leq i_{1}, \ldots, i_{k} \leq d}\left\|X_{i_{1}} \ldots X_{i_{k}}\right\|^{1 / k} .
$$

We define the outer spectral radius, which is defined via the formula

$$
\hat{\rho}\left(X_{1}, \ldots, X_{d}\right)=\sqrt{\rho\left(\sum \overline{X_{i}} \otimes X_{i}\right)}
$$

where $A \otimes B$ is just the usual Kronecker product and $\bar{A}$ is the complex conjugate of $A$. The outer spectral radius is essentially a special case of the the Blondel-Nesterov approximation of the joint spectral radius in 3. Moreover, the outer spectral radius relates to the quantum information theory literature as spectral radius of the completely positive $\operatorname{map} \phi(H)=\sum X_{i} H X_{i}^{*}$ [25, 15, 14, 21, 20, 8]. Under this guise, although in an independent fashion, a Rota-Strang type theory has been developed by G. Popescu for the outer spectral radius [20]. One goal will be to give a Gelfand type theorem, unify the various manifestations of the outer spectral radius and give an elementary treatment of the Popescu-Rota-Strang theory. Moreover, we will give a detailed spectral analysis of $\sum \overline{X_{i}} \otimes X_{i}$. We note that, up to this point in time, it does not appear that the connection between the Blondel-Nesterov relaxation of the joint spectral radius, the dynamics of completely positive maps and the Popescu theory of row contractions had been noticed.

Although the outer spectral radius is interesting in its own right as a natural relaxation of the joint spectral radius, the immediate question 
is: what dynamics does it describe? It turns out there is a satisfying answer here as well- it describes the dynamics of the iterates of a completely positive map. (This was the approach taken by Popescu in [20].) Moreover, the same tools used to analyze the outer spectral radius can be used to do a more detailed analysis of $\sum \overline{X_{i}} \otimes X_{i}$ itself. The "sinks" in terms of the dynamics here turn out to be ideals in the algebra generated by $X_{1}, \ldots, X_{d}$. In the special case that the completely positive map is trace preserving, sometimes referred to a quantum channel, the dynamics are particularly nice.

We can characterize the outer spectral radius $\hat{\rho}\left(X_{1}, \ldots, X_{d}\right)$ in terms of a Gelfand type formula.

Theorem 1.1. Let $\left(X_{1}, \ldots, X_{d}\right) \in M_{n}(\mathbb{C})^{d}$.

$$
\hat{\rho}\left(X_{1}, \ldots, X_{d}\right)=\lim _{k \rightarrow \infty} \sup _{\sum\left|a_{i_{1}, \ldots, i_{k}}\right|^{2}=1}\left\|\sum_{1 \leq i_{1}, \ldots, i_{k} \leq d} a_{i_{1}, \ldots, i_{k}} X_{i_{1}} \ldots X_{i_{k}}\right\|^{1 / k} .
$$

Theorem 1.1 is proven Section 3 .

Let $T$ be a square matrix. We call the subset of the eigenvalues of $T$ with maximum modulus which have maximal degeneracy index the maximal spectrum of $T$. If the maximal degeneracy index is 1 we say the maximal spectrum is nondegenerate. (The degeneracy index of an eigenvalue is the maximum size of the Jordan blocks correspond to that eigenvalue.) A canonical choice of positive $L$ such that $L-\sum X_{i} L X_{i}^{*}$, obtained in Theorem [1.9, our treatment of the Popescu's Rota-Strang theory, has a special enough form to imply that the maximal eigenvalue of $\sum \overline{X_{i}} \otimes X_{i}$ can be chosen to be non-negative and real-valued. (This is similar to the degenerate case of the classical Perron-Frobenius theorem, and can be viewed as a degenerate case of the Quantum Perron-Frobenius theorem[8].) That is, be have the following result.

Theorem 1.2 (Degenerate Quantum Perron-Frobenius theorem). Let $\left(X_{1}, \ldots, X_{d}\right) \in M_{n}(\mathbb{C})^{d}$. Let $T=\sum \overline{X_{i}} \otimes X_{i}$. There is a non-negative real eigenvalue $\lambda$ of $T$ with degeneracy index $\eta$, such that any other eigenvalue $\lambda^{\prime}$ of $T$ with degeneracy index $\eta^{\prime}$ has the property that either

(1) $\lambda=\left|\lambda^{\prime}\right|$ and $\eta \geq \eta^{\prime}$,

(2) $\lambda>\left|\lambda^{\prime}\right|$.

That is, the maximal spectrum has a real nonnegative element.

Theorem [1.2 is proven in Section [5.1. We give some further comments on the structure of the spectrum of $T$ in 5.3, which explains some observations by Blondel-Nesterov and the relationship with the Parrilo-Jadbabaie relaxation of the joint spectral radius. 


\subsection{Dynamics of quantum channels and other completely pos-} itive maps. The classical joint spectral radius can be seen as descibing the dynamics of switched linear systems [7, 2]. In parallel, we can use the outer spectral radius theory to understand the dynamics of completely positive maps, and more specifically the dynamics of quantum channels, for which there has been some recent interest [25, 15, 14, 21, 20, 8]. That is we want to understand the iterates of a $\operatorname{map} \phi: M_{n}(\mathbb{C}) \rightarrow M_{n}(\mathbb{C})$ of the form

$$
\phi(H)=\sum A_{i} H A_{i}^{*}
$$

by the Choi-Kraus theorem [6, 12, the quantum channels are those completely positive maps such that additionally $\sum A_{i}^{*} A_{i}=I$. These are the trace preserving completely positive maps. Maps satisfying $\sum A_{i} A_{i}^{*}=I$ are called unital. Given $T$ of the form $\sum \overline{A_{i}} \otimes A_{i}$, we can write the map $\phi_{T}(H)=\sum A_{i} H A_{i}^{*}$. (Moreover by the Choi-Kraus characterization, this is all of them.) Note that

$$
\phi_{T_{1} T_{2}}=\phi_{T_{1}} \circ \phi_{T_{2}} .
$$

Therefore, the dynamics of the map $\phi_{T}$ are essentially those of $T^{n}$. Therefore, we can apply the typical idea in dynamics and study the part of $T$ corresponding to the maximal spectrum.

1.1.1. The general case. Let $T=\sum \overline{X_{i}} \otimes X_{i}$. Additionally, assume that $\hat{\rho}\left(X_{1}, \ldots, X_{d}\right)=1$. Let $m_{T}$ denote the cardinality of the maximal spectrum of $T$. We define $\Lambda$ to be the closure of the subgroup of the torus $\mathbb{T}^{m_{T}}$ generated by the point $\tau=\left(\lambda_{1}, \ldots, \lambda_{m_{T}}\right)$ where the $\lambda_{i}$ are the elements of the maximal spectrum. As a topological space and a group, $\Lambda \cong \mathbb{T}^{c_{T}} \times H$ where $H$ is some finite abelian group and $c_{T} \in \mathbb{N}$. There is natural bijective map $T_{\lambda}$ from $\Lambda$ to the set of limit points of the sequence $\frac{T^{n}}{\left\|T^{n}\right\|}$, since along any sequence such that $\tau^{n}$ converges, the $\frac{T^{n}}{\left\|T^{n}\right\|}$ converges. (One can see this by considering the Jordan canonical form.) Moreover, note that either $T_{\lambda}^{2}=T_{\lambda^{2}}$ or $T_{\lambda}^{2}=0$, depending on whether or not the maximal spectrum is nondegenerate. Now by calculation,

$$
T T_{\lambda}=T_{\lambda} T=T_{\tau \lambda}
$$

The set of $T_{\lambda}$ describe the asymptotic dynamics of $T^{n}$. Now,

$$
\hat{T}=\int_{\Lambda} T_{\lambda} \mathrm{d} \lambda
$$


where the integral is taken with respect to normalized Haar measure on $\Lambda$. Alternatively, we could take the elementary and equivalent definition

$$
\hat{T}=\lim _{N \rightarrow \infty} \frac{1}{N} \sum_{n=1}^{N} \frac{T^{n}}{\left\|T^{n}\right\|},
$$

as for large $n$, the quantity $\frac{T^{n}}{\left\|T^{n}\right\|}$ behaves like a random $T_{\lambda}$. The matrix $\hat{T}$ essentially describes the average asymptotic dynamics. Note that either $\hat{T}^{2}=\hat{T}$ or $\hat{T}^{2}=0$.

Theorem 1.3. Let $T=\sum \overline{X_{i}} \otimes X_{i}$. Additionally, assume that $\hat{\rho}\left(X_{1}, \ldots, X_{d}\right)=$ 1 .

(1) There is an $a \in \mathbb{N}$ such that for every $\lambda \in \Lambda$ there are $A_{\lambda, 1} \ldots A_{\lambda, a} \in$ $M_{n}(\mathbb{C})$ such that $T_{\lambda}=\sum_{j=1}^{a} \overline{A_{\lambda, j}} \otimes A_{\lambda, j}$.

(2) There are $B_{k} \in M_{n}(\mathbb{C})$ whose span is a nonzero ideal in the algebra generated by $X_{1}, \ldots, X_{d}$ such that $\hat{T}=\sum_{k} \overline{B_{k}} \otimes B_{k}$. Moreover, the $A_{\lambda, j}$ are contained in the span of the $B_{k}$ 's.

Theorem 1.3 is proved as Proposition 5.2

We can interpret the above theorem as saying that a sink in this framework is somehow corresponded to an ideal $I$ spanned by the $B_{k}$ 's. Moreover, this ideal is weakly graded into the subspaces $I_{\lambda}$ spanned by $A_{\lambda, j}$ in the sense that the sum of all the $I_{\lambda}$ is $I$, and $\Lambda$ naturally acts on the $I_{\lambda}$. Note that

$$
T T_{\lambda}=T_{\tau \lambda}=\sum_{i=1}^{d} \sum_{j=1}^{a} \overline{X_{i} A_{\lambda, j}} \otimes X_{i} A_{\lambda, j}=\sum_{j=1}^{a} \overline{A_{\tau \lambda, j}} \otimes A_{\tau \lambda, j} .
$$

That is, the movement of $I_{\lambda}$ as $\lambda$ ranges through $\Lambda$ somehow should be thought of as an asymptotic orbit. Note these dynamics describe the limiting coefficients of the channel $\phi_{T^{n} /\left\|T^{n}\right\|}$ and not the range of such a channel. In the case where $T_{\lambda}^{2}=0$, the ideal $I$ must satisfy that $I^{2}=0$. Moreover, whenever the maximal spectrum contains a single element, as happens generically, the group $\Lambda$ has a single element, and therefore $\hat{T}=T_{\lambda}$, which in turn should be thought of as saying the action has no circulation in the limit or is ergodic.

Whenever $X_{1}, \ldots, X_{d}$ generate the full algebra of $n$ by $n$ matrices, average asymptotic dynamics simplify. The condition that the coordinates generate the full algebra essentially corresponds to the condition of having all positive entries in the classical Perron-Frobenius theorem. Under an irreducibility type assumptions various Quantum Perron-Frobenius theorems which establish the existence of a simple 
real eigenvalue with maximum modulus have been obtained by EvansHoegh-Krohn[8], Schrader [26] and Lagro-Yang-Xiong[13]. We essentially gather some more detailed structure than the aforementioned works in the finite dimensional case by applying Theorem 1.3 .

Theorem 1.4 (Quantum Perron-Frobenius Theorem). Let $T=\sum \overline{X_{i}} \otimes$ $X_{i}$. Additionally, assume that $\hat{\rho}\left(X_{1}, \ldots, X_{d}\right)=1$. If $X_{1}, \ldots, X_{d}$ generate the full algebra of $n$ by $n$ matrices, then $\hat{T}$ has rank 1 . Moreover, in such a case, $\hat{T}=\sum_{k} \overline{B_{k}} \otimes B_{k}$ where the $B_{k}$ 's span $M_{n}(\mathbb{C})$. That is, the ideal described in Theorem 1.3 is equal to the whole algebra, as the only nonzero ideal in $M_{n}(\mathbb{C})$ is $M_{n}(\mathbb{C})$ itself.

Theorem 1.4 is proved as Proposition 5.4. Corollary 5.5 establishes that whenever the $X_{i}$ 's generate the full algebra, then it can be conjugated to a row co-isometry or column isometry, therefore the corresponding conjugated channels are unital and trace preserving respectively.

1.1.2. Nondegenerate maximal spectrum and quantum channels. If the maximal spectrum is nondegenerate, the situation simiplifies somewhat. Firstly, $T_{\lambda} T_{\lambda^{\prime}}=T_{\lambda \lambda^{\prime}}$. That is, the map taking $\lambda$ to $T_{\lambda}$ behaves essentially like a group homomorphism. (However, it is possible that $T_{\lambda}$ may have a nonzero kernel.) We also note that if $T$ describes a quantum channel, then $T$ must have nondegenerate maximal spectrum, as otherwise, there would be a positive $H$ such that $\left\|\phi_{T^{n}}(H)\right\| \rightarrow \infty$. However, this cannot happen as $\phi_{T}$ is trace preserving and a completely positive map.

1.2. The outer spectral radius formula and the Blondel-Nesterov approximation. We will now discuss how Theorem 1.1 can be used to exhibit some well known approximations of the joint spectral radius which originated with Blondel and Nesterov in [3], and has been improved in various ways in [27, 16].

Using Theorem 1.1 we can relate the outer spectral radius to the joint spectral radius. We leave the details of this calculation to the reader.

Corollary 1.5. Let $\left(X_{1}, \ldots, X_{d}\right) \in M_{n}(\mathbb{C})^{d}$.

$$
\frac{1}{\sqrt{d}} \hat{\rho}\left(X_{1}, \ldots, X_{d}\right) \leq \rho\left(X_{1}, \ldots, X_{d}\right) \leq \hat{\rho}\left(X_{1}, \ldots, X_{d}\right)
$$

That is, the outer spectral radius itself serves as an approximation of the joint spectral radius. We note that the outer spectral radius is relatively easy to compute, (since it is just the maximum modulus 
eigenvalue of an $n^{2}$ by $n^{2}$ matrix) whereas the joint spectral radius is somewhat difficult [4, 5], although there are several ways to approximate the joint spectral radius using techniques from optimization[3, 16, 1].

Blondel and Nesterov[3] showed that, for tuples of matrices with nonnegative entries,

$$
\rho\left(X_{1}, \ldots, X_{d}\right)=\lim _{k \rightarrow \infty} \rho\left(\sum X_{i}^{\otimes k}\right)^{1 / k} .
$$

Here $A^{\otimes n}$ denotes the $n$-th Kronecker power of $A$, the Kronecker product of $A$ with itself $n$ times. Moreover, they obtained inequalities as in 1.5. In [27], Xiao and Xu showed that, in general,

$$
\rho\left(X_{1}, \ldots, X_{d}\right)=\limsup _{k \rightarrow \infty} \rho\left(\sum X_{i}^{\otimes k}\right)^{1 / k} .
$$

We use the outer spectral radius to generalize the Blondel-Nesterov formula in a way that eliminates the supremum from the Xiao-Xu formula and gives bounds on the error which are the same as the error bounds in the Blondel-Nesterov [3] approximation. That is, we give a family of asymptotically tight approximations which converge to the joint spectral radius in Corollary 1.6 for arbitrary matrices over $\mathbb{C}$. Specifically,

$$
\begin{aligned}
\rho\left(X_{1}, \ldots, X_{d}\right) & =\lim _{k \rightarrow \infty} \hat{\rho}\left(X_{1}^{\otimes k}, \ldots, X_{d}^{\otimes k}\right)^{1 / k}, \\
& =\lim _{k \rightarrow \infty} \rho\left(\sum \overline{X_{i}^{\otimes k}} \otimes X_{i}^{\otimes k}\right)^{1 / 2 k} .
\end{aligned}
$$

In fact, Xiao and $\mathrm{Xu}[27]$ noted that, even for real matrices, $\lim _{k \rightarrow \infty} \rho\left(\sum X_{i}^{\otimes k}\right)^{1 / k}$ may not exist. We can now, in light of our new approximation, view this phenomenon as stemming from the fact that $k$ could be odd, since, over the reals, our formula implies that $\lim _{k \rightarrow \infty} \rho\left(\sum X_{i}^{\otimes 2 k}\right)^{1 / 2 k}$ exists.

To obtain our formula, we observe that

$$
\rho\left(X_{1}^{\otimes k}, \ldots, X_{d}^{\otimes k}\right)=\rho\left(X_{1}, \ldots, X_{d}\right)^{k} .
$$

So, we obtain a Blondel-Nesterov type formula for the joint spectral radius as a consequence of Corollary 1.5.

Corollary 1.6. Let $\left(X_{1}, \ldots, X_{d}\right) \in M_{n}(\mathbb{C})^{d}$.

$$
\frac{1}{\sqrt[2 k]{d}} \hat{\rho}\left(X_{1}^{\otimes k}, \ldots, X_{d}^{\otimes k}\right)^{1 / k} \leq \rho\left(X_{1}, \ldots, X_{d}\right) \leq \hat{\rho}\left(X_{1}^{\otimes k}, \ldots, X_{d}^{\otimes k}\right)^{1 / k} .
$$

Namely,

$$
\rho\left(X_{1}, \ldots, X_{d}\right)=\lim _{k \rightarrow \infty} \hat{\rho}\left(X_{1}^{\otimes k}, \ldots, X_{d}^{\otimes k}\right)^{1 / k} .
$$




\subsection{A Popescu-Rota-Strang theory of the outer spectral ra-} dius. We can obtain an analogue of a theorem on the joint spectral radius of Rota and Strang[22, 23] which states that:

$$
\rho\left(X_{1}, \ldots, X_{d}\right)=\inf _{N \in \mathfrak{N}} \sup _{i}\left\|X_{i}\right\|_{N}
$$

where $\mathfrak{N}$ is the set of all consistent matrix norms on $M_{n}(\mathbb{C})$. The outer spectral radius is the infimum over all "two norms" of the block matrix $\left[\begin{array}{lll}X_{1} & \ldots & X_{d}\end{array}\right]$, which can be made formal as the infimum over all points simultaneously similar to $\left(X_{1}, \ldots, X_{d}\right)$ of the norm as a block matrix. A version of the Rota-Strang theory was developed by Popescu in [20] for the row ball viewed the set of Kraus coefficients of iterable contractive completely positive maps which under translation becomes a Rota-Strang theory of the outer spectral radius initiated by BlondelNesterov. (Apparently, that the two quantities were the same had not been realized.) One of our goals will be to give an elementary treatment of Popescu's Rota-Strang theory for the outer spectral radius on its own terms.

Theorem 1.7 (Popescu [20]). Let $\left(X_{1}, \ldots, X_{d}\right) \in M_{n}(\mathbb{C})^{d}$.

$$
\hat{\rho}\left(X_{1}, \ldots, X_{d}\right)=\inf _{S \in G L_{n}(\mathbb{C})}\left\|\left[\begin{array}{llll}
S X_{1} S^{-1} & \ldots & S X_{d} S^{-1}
\end{array}\right]\right\|_{2} .
$$

Theorem 1.7 follows directly from Theorem 1.9 .

We also note the following observation, which follows directly from the Rota-Strang theory, or perhaps even the classical Jordan decomposition.

Theorem 1.8. Let $\left(X_{1}, \ldots, X_{d}\right) \in M_{n}(\mathbb{C})^{d}$.

$$
\hat{\rho}\left(X_{1}, \ldots, X_{d}\right)=\inf _{N \in \mathfrak{N}}\left\|\sum \overline{X_{i}} \otimes X_{i}\right\|_{N}
$$

A tuple $\left(X_{1}, \ldots, X_{d}\right) \in M_{n}(\mathbb{C})^{d}$ is called a row contraction if the block matrix $\left[\begin{array}{lll}X_{1} & \ldots & X_{d}\end{array}\right]$ has 2 -norm strictly less than 1 . Row contractions have been extensively studied for their dilation theoretic properties. (e.g [9, 18, 19, 10]) Row contractions are known to satify the following inequality[18]:

$$
\sup _{\sum \mid a_{i_{1}, \ldots,\left.i_{k}\right|^{2}=1}}\left\|\sum_{1 \leq i_{1}, \ldots, i_{k} \leq d} a_{i_{1}, \ldots, i_{k}} X_{i_{1}} \ldots X_{i_{k}}\right\|<1 .
$$

The inequality above gives a hint of the connection of row contractions and outer spectral radius, given the outer spectral radius formula in Theorem 1.1.

The outer spectral radius is characterized in terms of a Lyapunov type condition and equivalence with row contractions. 
Theorem 1.9 (Popescu [20]). Let $\left(X_{1}, \ldots, X_{d}\right) \in M_{n}(\mathbb{C})^{d}$. The following are equivalent:

(1) $\hat{\rho}\left(X_{1}, \ldots, X_{d}\right)<1$.

(2) There is a positive definite matrix $L$ such that $L-\sum X_{i} L X_{i}^{*}$ is positive definite.

(3) There is $S \in G L_{n}(\mathbb{C})$ such that $\left(S X_{1} S^{-1}, \ldots, S X_{d} S^{-1}\right)$ is a row contraction.

Theorem 1.9 is proved in several parts. The implication $(1 \Rightarrow 2)$ is part 3 of Proposition 4.1 which explicitly constructs a canonical rationally computable choice for $L$ for which $L-\sum X_{i} L X_{i}^{*}=I$. The implication $(3 \Rightarrow 1)$ follows from Proposition 3.1. The equivalence $(2 \Rightarrow 3)$ follows by letting $S=L^{-1 / 2}$ and $(3 \Rightarrow 2)$ by setting $L=$ $\left(S^{*} S\right)^{-1}$.

From the point of view of free analysis or noncommutative function theory, Theorem 1.9 is interesting because it shows that any map defined on the set of row contractions extends to all outer spectral contractions, which includes other domains of interest such as the column contractions. (The author was originally concerned with these kind of considerations.)

\section{Preliminaries}

We fix the notation that $I$ is the identity matrix in $M_{n}(\mathbb{C})$ and 1 is the identity matrix in $M_{n^{2}}(\mathbb{C})$.

We will use the following matrix ordering: for two self-adjoint matrices $A, B \in M_{m}(\mathbb{C})$, we say that $A \leq B$ if $B-A$ is positive semidefinite and we say that $A<B$ if $B-A$ is positive definite. Notably, the notation $A>0$ means that $A$ is positive definite and $A \geq 0$ means that $A$ is positive semidefinite.

We adopt a "vertical tensor notation" to conserve space and enhance visual symmetry during calculations:

$$
\underset{B}{\stackrel{A}{\otimes}}=A \otimes B .
$$

To proceed we will need to define and describe two important maps: the $\psi$ involution and the partial trace $Q$.

2.1. The $\psi$ involution. The $\psi$ involution is the linear map $\psi$ : $M_{n^{2}}(\mathbb{C}) \rightarrow M_{n^{2}}(\mathbb{C})$ defined by the relations

$$
\psi\left(E_{i+(j-1) n, k+(l-1) n}\right)=E_{i+(k-1) n, j+(l-1) n}
$$

where $E_{a, b}$ is the matrix with a 1 at the $(a, b)$ entry and 0 everywhere

else. Informally, the $\psi$ involution swaps the positions of $j$ and $k$. Since 
$E_{a, b}$ form a basis for $M_{n^{2}}(\mathbb{C})$ we can extend the map $\psi$ by linearity to all of $M_{n^{2}}(\mathbb{C})$. We formally adopt the notation

$$
A^{\psi}=\psi(A) \text {. }
$$

The vectorization map is the linear map vec $: M_{n}(\mathbb{C}) \rightarrow \mathbb{C}^{n^{2}}$ defined by the relations

$$
\operatorname{vec} E_{i, j}=e_{i+(j-1) n}
$$

where $e_{k}$ is the $k$-th elementary basis vector of $\mathbb{C}^{n^{2}}$. We extend vec to all of $M_{n}(\mathbb{C})$ by linearity. The vectorization map is used throughout matrix theory, see [11].

The $\psi$ involution will be very useful to us in our analysis of the outer spectral radius and row contractions. It is especially useful given the following proposition, which shows that the $\psi$ involution has a rich algebraic structure. The $\psi$ involution was used to develop algorithms for understanding finite dimensional matrix algebras [17].

Proposition 2.1. The map $\psi$ satisfies the following properties for any $A, B, C, D \in M_{n}(\mathbb{C})$ and $E, F \in M_{n^{2}}(\mathbb{C})$ :

The map $\psi$ is an involution:

$$
\left[E^{\psi}\right]^{\psi}=E \text {. }
$$

The map $\psi$ takes twisted tensor products to outer products:

$$
\left(\begin{array}{l}
\bar{A} \\
\stackrel{\otimes}{B}
\end{array}\right)^{\psi}=(\operatorname{vec} B)(\operatorname{vec} A)^{*}
$$

\section{4-modularity:}

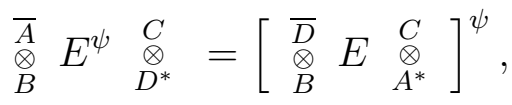

Schur Product Property:

$$
\begin{gathered}
E \geq 0, F \geq 0 \Rightarrow\left[E^{\psi} F^{\psi}\right]^{\psi} \geq 0 . \\
\text { (Here } G \geq 0 \text { means that } G \text { is positive semidefinite.) }
\end{gathered}
$$

The proof of Proposition 2.1 is elementary and left to the reader. (For the first three items, it is enough to check the identity on elementary matrices. For the Schur product property, it is enough to check the inequality when $E$ and $F$ are rank 1.)

We note that the Schur Product Property is particularly interesting since it provides a multiplication on $M_{n^{2}}(\mathbb{C})$ which preserves positivity, much like the classical Schur product. (The Schur product is also known as the Hadamard product, or entry-wise multiplication.) 
In fact, the Schur Product Property can actually be used to prove the Schur product theorem for the classical Schur product. Consider the map $\tau: M_{n}(\mathbb{C}) \rightarrow M_{n^{2}}(\mathbb{C})$ defined by

$$
\tau\left(E_{i, j}\right)=\stackrel{\substack{E_{i, j} \\ E_{i, j}}}{E_{i}}
$$

One can show that $A \geq 0$ if and only if $\tau(A) \geq 0$, and, moreover, one can show that

$$
A \circ B=\tau^{-1}\left(\left[\tau(A)^{\psi} \tau(B)^{\psi}\right]^{\psi}\right)
$$

where $A \circ B$ denotes the classical Schur product.

We also note that the properties of taking twisted tensor product to outer products and 4-modularity are equivalent to the definition of the $\psi$ involution up to multiplication by a constant.

2.2. The partial trace $Q$. Another useful map for us will be the partial trace $Q$ which is defined to be the map $Q: M_{n^{2}}(\mathbb{C}) \rightarrow M_{n}(\mathbb{C})$ which satisfies

$$
Q\left(E_{i+n(j-1), k+n(l-1)}\right)=\chi_{j l} E_{i, k} .
$$

where $\chi_{a b}$ equals 1 if $a=b$ and 0 otherwise.

Proposition 2.2. The map $Q$ satisfies the following properties for any $A, B \in M_{n}(\mathbb{C})$ and $E, F \in M_{n^{2}}(\mathbb{C})$ :

The $\psi$-identity identity:

$$
Q\left([1]^{\psi}\right)=I .
$$

The vectorization identity:

$$
Q\left([\operatorname{vec} A][\operatorname{vec} B]^{*}\right)=A B^{*} \text {. }
$$

Modularity:

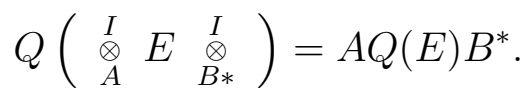

The map $Q$ is positive:

$$
E \geq 0 \Rightarrow Q(E) \geq 0
$$

\section{Product property:}

$$
Q(E) \geq 0, F \geq 0 \Rightarrow Q\left(\left[F^{\psi} E^{\psi}\right]^{\psi}\right) \geq 0 .
$$

We leave the above properties as an exercise to the reader. 


\section{The OUTER SPECTRAL RADIUS FORMUlA}

Now we show the outer spectral radius formula given as Theorem 1.1 .

Proof of Theorem 1.1. Recall

$$
\hat{\rho}\left(X_{1}, \ldots, X_{d}\right)=\sqrt{\rho\left(\sum \begin{array}{c}
\overline{X_{i}} \\
\otimes \\
X_{i}
\end{array}\right)} .
$$

Let $W=\sum \begin{gathered}\overline{X_{i}} \\ \otimes \\ X_{i}\end{gathered}$. By the classical spectral radius formula, it is enough to calculate

$$
\sqrt{\lim _{k \rightarrow \infty}\left\|W^{k}\right\|_{F}^{1 / k}}
$$

where $\|\cdot\|_{F}$ denotes the Frobenius norm. Note that

$$
W^{k}=\sum_{1 \leq i_{1}, \ldots, i_{k} \leq d} \begin{gathered}
\overline{X_{i_{1}} \ldots X_{i_{k}}} \\
X_{i_{1} \ldots X_{i_{k}}}^{\otimes}
\end{gathered}
$$

So, applying Proposition 2.1,

$$
\left(W^{k}\right)^{\psi}=\sum_{1 \leq i_{1}, \ldots, i_{k} \leq d}\left(\operatorname{vec} X_{i_{1}} \ldots X_{i_{k}}\right)\left(\operatorname{vec} X_{i_{1}} \ldots X_{i_{k}}\right)^{*}
$$

Let $V_{k}$ be the $n^{2}$ by $d^{k}$ with columns vec $X_{i_{1}} \ldots X_{i_{k}}$. Observe that

$$
\begin{aligned}
\left\|V_{k}\right\|_{2} & =\sup _{\sum\left|a_{i_{1}, \ldots, i_{k}}\right|^{2}=1}\left\|\sum_{1 \leq i_{1}, \ldots, i_{k} \leq d} a_{i_{1}, \ldots, i_{k}} \operatorname{vec} X_{i_{1}} \ldots X_{i_{k}}\right\|, \\
& =\sup _{\sum\left|a_{i_{1}, \ldots, i_{k}}\right|^{2}=1}\left\|\sum_{1 \leq i_{1}, \ldots, i_{k} \leq d} a_{i_{1}, \ldots, i_{k}} X_{i_{1}} \ldots X_{i_{k}}\right\|_{F} .
\end{aligned}
$$

Note $V_{k} V_{k}^{*}=\left(W^{k}\right)^{\psi}$. So

$$
\left\|\left(W^{k}\right)^{\psi}\right\|_{2}=\sup _{\sum\left|a_{i_{1}, \ldots, i_{k}}\right|^{2}=1}\left\|\sum_{1 \leq i_{1}, \ldots, i_{k} \leq d} a_{i_{1}, \ldots, i_{k}} X_{i_{1}} \ldots X_{i_{k}}\right\|_{F}^{2} .
$$

Observe $\left\|\left(W^{k}\right)^{\psi}\right\|_{F}=\left\|W^{k}\right\|_{F}$ and, so,

$$
\left\|\left(W^{k}\right)^{\psi}\right\|_{2} \leq\left\|W^{k}\right\|_{F} \leq n\left\|\left(W^{k}\right)^{\psi}\right\|_{2} .
$$


Namely, we see that

$$
\begin{aligned}
\lim _{k \rightarrow \infty}\left\|W^{k}\right\|_{F}^{1 / 2 k} & =\lim _{k \rightarrow \infty}\left\|\left(W^{k}\right)^{\psi}\right\|_{2}^{1 / 2 k} \\
& =\lim _{k \rightarrow \infty} \sup _{\sum\left|a_{i_{1}, \ldots, i_{k}}\right|^{2}=1}\left\|\sum_{1 \leq i_{1}, \ldots, i_{k} \leq d} a_{i_{1}, \ldots, i_{k}} X_{i_{1}} \ldots X_{i_{k}}\right\|_{F}^{1 / k} .
\end{aligned}
$$

We now prove a proposition which immediately implies $(3 \Rightarrow 1)$ from Theorem 1.9 when combined with the observation that for any $S \in G L_{n}(\mathbb{C})$,

$$
\hat{\rho}\left(X_{1}, \ldots, X_{d}\right)=\hat{\rho}\left(S X_{1} S^{-1}, \ldots, S X_{d} S^{-1}\right) .
$$

Proposition 3.1. If $\left(X_{1}, \ldots, X_{d}\right) \in M_{n}(\mathbb{C})^{d}$ is a row contraction, then $\hat{\rho}\left(X_{1}, \ldots, X_{d}\right)<1$.

Proof. Let $W$ be as in the proof of Theorem 1.1. Since $\left(X_{1}, \ldots, X_{d}\right) \in$ $M_{n}(\mathbb{C})^{d}$ is a row contraction, $\sum X_{i} X_{i}^{*}<1$. So, $\sum X_{i} X_{i}^{*} \leq t$ for some $0<t<1$. Note $Q\left(W^{\psi}\right)=\sum X_{i} X_{i}^{*}$. So $Q\left([t-W]^{\psi}\right) \geq 0$, so, since $\left[W^{k}\right]^{\psi} \geq 0$, by the product property of $Q$,

$$
Q\left(\left[t W^{k}-W^{k+1}\right]^{\psi}\right)=Q\left(\left[W^{k}(t-W)\right]^{\psi}\right) \geq 0 .
$$

That is,

$$
Q\left(\left[W^{k+1}\right]^{\psi}\right) \leq t Q\left(\left[W^{k}\right]^{\psi}\right) .
$$

Inductively, $Q\left(\left[W^{k}\right]^{\psi}\right) \leq t^{k} Q\left(1^{\psi}\right)=t^{k}$. Let vec $u_{1}\left(X_{1}, \ldots, X_{d}\right)$ be an eigenvector corresponding to the maximum eigenvalue of $\left(W^{k}\right)^{\psi}$ normalized such that

$$
u_{1}\left(X_{1}, \ldots, X_{d}\right)=\sum_{1 \leq i_{1}, \ldots, i_{k} \leq d} a_{i_{1}, \ldots, i_{k}} X_{i_{1}} \ldots X_{i_{k}}
$$

where $\sum_{1 \leq i_{1}, \ldots, i_{k} \leq d}\left|a_{i_{1}, \ldots, i_{k}}\right|^{2}=1$. Note that

$$
\left\|\sum_{1 \leq i_{1}, \ldots, i_{k} \leq d} a_{i_{1}, \ldots, i_{k}} X_{i_{1}} \ldots X_{i_{k}}\right\|_{F}
$$

is maximized subject to the constraint $\sum_{1 \leq i_{1}, \ldots, i_{k} \leq d}\left|a_{i_{1}, \ldots, i_{k}}\right|^{2}=1$ by $u_{1}$. Note,

$$
\left(\operatorname{vec} u_{1}\left(X_{1}, \ldots, X_{d}\right)\right)\left(\operatorname{vec} u_{1}\left(X_{1}, \ldots, X_{d}\right)\right)^{*} \leq\left(W^{k}\right)^{\psi} \text {. }
$$

Applying $Q$, using the vectorization identity and the positivity of $Q$ from Proposition 2.2, we get that

$$
\begin{aligned}
u_{1}\left(X_{1}, \ldots, X_{d}\right) u_{1}\left(X_{1}, \ldots, X_{d}\right)^{*} & \leq Q\left(\left[W^{k}\right]^{\psi}\right) \\
& \leq t^{k}
\end{aligned}
$$


Now, we get that

$$
\begin{aligned}
\hat{\rho}\left(X_{1}, \ldots, X_{d}\right) & =\lim _{k \rightarrow \infty} \sup _{\sum\left|a_{i_{1}, \ldots, i_{k}}\right|^{2}=1}\left\|\sum_{1 \leq i_{1}, \ldots, i_{k} \leq d} a_{i_{1}, \ldots, i_{k}} X_{i_{1}} \ldots X_{i_{k}}\right\|^{1 / k} \\
& \leq \lim _{k \rightarrow \infty}\left(t^{k}\right)^{1 / k} \\
& =t \\
& <1 .
\end{aligned}
$$

So, by Theorem 1.1 we are done.

\section{Construction of the Lyapunov matrix}

We will now begin work towards showing the implication $(1 \Rightarrow 2)$ Theorem 1.9, which we will accomplish through an algebraic construction of the matrix $L$.

Let $\left(X_{1}, \ldots, X_{d}\right) \in M_{n}(\mathbb{C})^{d}$ such that $\hat{\rho}\left(X_{1}, \ldots, X_{d}\right)<1$. We define the elementary Pick matrix corresponding to $\left(X_{1}, \ldots, X_{d}\right)$ to be

$$
P=\left[\left(1-\sum \begin{array}{c}
\overline{X_{i}} \\
\otimes \\
X_{i}
\end{array}\right)^{-1}\right]^{\psi}
$$

In [17], it was shown that the rank of $P$ gives the dimension of the algebra generated by the $X_{i}$ and, in fact, that the columns of $P$ span that algebra. We establish some basic facts about the elementary Pick matrix which culminate in item $(3)$ which gives implication $(1 \Rightarrow 2)$ in Theorem 1.9.

Proposition 4.1. Let $\left(X_{1}, \ldots, X_{d}\right) \in M_{n}(\mathbb{C})^{d}$ such that $\hat{\rho}\left(X_{1}, \ldots, X_{d}\right)<$ 1 and $P$ be the corresponding elementary Pick matrix. The following are true:

(1) $P \geq 0$,

(2) $P-\sum \underset{X_{i}}{\stackrel{I}{\otimes}} P \underset{X_{i}^{*}}{\stackrel{I}{\otimes}}=1^{\psi} \geq 0$,

(3) Let $L=Q(P) . \stackrel{L}{L}>0$ and

$$
L-\sum X_{i} L X_{i}^{*}=I>0 .
$$

Proof. (1) Let

$$
S=\left(\sum \begin{array}{c}
\overline{X_{i}} \\
\stackrel{\otimes}{X_{i}}
\end{array}\right)^{\psi}
$$

We first note that

$$
S=\sum\left(\operatorname{vec} X_{i}\right)\left(\operatorname{vec} X_{i}\right)^{*}
$$


since $\psi$ has the property that it takes twisted tensor products to outer products as was established in Proposition 2.1. Namely, $S \geq 0$ since it is a sum of positive semidefinite rank one matrices. Note that, since $\hat{\rho}\left(X_{1}, \ldots, X_{d}\right)<1$ implies that the spectral radius of $S^{\psi}$ is less than one, we can expand $P$ using the geometric series:

$$
\begin{aligned}
P & =\left[\left(1-\sum \begin{array}{c}
\overline{X_{i}} \\
\otimes \\
X_{i}
\end{array}\right)^{-1}\right]^{\psi} \\
& =\left[\left(1-S^{\psi}\right)^{-1}\right]^{\psi} \\
& =\left[\sum_{k=0}^{\infty}\left(S^{\psi}\right)^{k}\right]^{\psi} . \\
& =\sum_{k=0}^{\infty}\left[\left(S^{\psi}\right)^{k}\right]^{\psi}
\end{aligned}
$$

We note that each term $\left[\left(S^{\psi}\right)^{k}\right]^{\psi} \geq 0$ by the Schur Product Property in Proposition 2.1, so we get that

$$
P=\sum_{k=0}^{\infty}\left[\left(S^{\psi}\right)^{k}\right]^{\psi} \geq 0
$$

and we are done.

(2) Now we want to show that

$$
P-\sum \stackrel{I}{\stackrel{\otimes}{\otimes}} P \underset{X_{i}}{\stackrel{I}{\otimes}} \underset{X_{i}^{*}}{\stackrel{P}{*}} \geq 0
$$

First we note that

$$
\begin{aligned}
& P-\sum \underset{X_{i}}{\stackrel{I}{\otimes}} P \underset{X_{i}^{*}}{\stackrel{I}{\otimes}}=P-\sum \underset{X_{i}}{\stackrel{I}{\otimes}}\left[\left(1-\sum \begin{array}{c}
\overline{X_{i}} \\
\stackrel{\otimes}{\otimes} \\
X_{i}
\end{array}\right)^{-1}\right]^{\psi} \underset{X_{i}^{*}}{\stackrel{I}{\otimes}} \\
& =P-\left[\sum \begin{array}{c}
\overline{X_{i}} \\
\otimes \\
X_{i}
\end{array}\left(1-\sum \begin{array}{c}
\overline{X_{i}} \\
\otimes \\
X_{i}
\end{array}\right)^{-1}\right]^{\psi}
\end{aligned}
$$


by the 4-modularity of $\psi$ from Proposition 2.1. Now we see that

$$
\begin{aligned}
& P-\sum \underset{X_{i}}{\stackrel{I}{\otimes}} P{ }_{X_{i}^{*}}^{\stackrel{I}{\otimes}}=P-\left[\sum \begin{array}{c}
\overline{X_{i}} \\
\stackrel{\otimes}{X_{i}}
\end{array}\left(1-\sum \begin{array}{c}
\overline{X_{i}} \\
\stackrel{\otimes}{X_{i}}
\end{array}\right)^{-1}\right]^{\psi} \\
& =\left[\left(1-\sum \begin{array}{c}
\overline{X_{i}} \\
\otimes \\
X_{i}
\end{array}\right)^{-1}\right]^{\psi}-\left[\sum \begin{array}{c}
\overline{X_{i}} \\
\otimes \\
X_{i}
\end{array}\left(1-\sum \begin{array}{c}
\overline{X_{i}} \\
\otimes \\
X_{i}
\end{array}\right)^{-1}\right]^{\psi} \\
& =\left[\left(1-\sum \begin{array}{c}
\overline{X_{i}} \\
\stackrel{\otimes}{X_{i}}
\end{array}\right)\left(1-\sum \begin{array}{c}
\overline{X_{i}} \\
\otimes \\
X_{i}
\end{array}\right)^{-1}\right]^{\psi} \\
& =[1]^{\psi} \\
& =(\operatorname{vec} I)(\operatorname{vec} I)^{*} \\
& \geq 0
\end{aligned}
$$

so we are done.

(3) Let $L=Q(P)$. We want to show that $L>0$ and

$$
L-\sum X_{i} L X_{i}^{*}>0 \text {. }
$$

Firstly, we note that

$$
L-\sum X_{i} L X_{i}^{*}=Q\left(P-\sum \underset{X_{i}}{\stackrel{I}{\otimes}} P{ }_{X_{i}^{*}}^{\stackrel{I}{\otimes}}\right),
$$

via the modularity of $Q$ in Proposition 2.2, Now,

$$
\begin{aligned}
L-\sum X_{i} L X_{i}^{*} & =Q\left(P-\sum \stackrel{I}{\otimes}_{X_{i}}^{I} P{ }_{X_{i}^{*}}^{\stackrel{I}{\otimes}}\right. \\
& =Q\left([1]^{\psi}\right), \\
& =I, \\
& >0 .
\end{aligned}
$$

Since $L=Q(P)$ and $P \geq 0$, we see that $L \geq 0$ by the positivity of $Q$ in Proposition 2.2. Now, $L \geq L-\sum X_{i} L X_{i}^{*}>0$, so we are done.

5. The outer SPECTRUm AND DyNAmiCs OF COMPletely POSITIVE MAPS

We now begin an endeavor to understand the spectral theory of

$$
T=\sum \overline{X_{i}} \otimes X_{i}
$$

The spectrum of $T$ can the though of as the outer spectrum of the tuple $\left(X_{1}, \ldots, X_{d}\right)$. Our analysis of the outer spectrum gives insight into the dynamics of completely positive maps as was described in the introduction. 
5.1. Maximum eigenvalue is nonnegative. First, we can quickly use our formula for the Lyapunov matrix from Proposition 4.1 to show that the spectral radius of $T$ is equal to the maximum eigenvalue of $T$.

Proposition 5.1. Let $X=\left(X_{1}, \ldots, X_{d}\right) \in M_{n}(\mathbb{C})^{d}$. Let $T=\sum \overline{X_{i}} \otimes$ $X_{i}$. There is a real nonnegative eigenvalue $\lambda$ of $T$ such that $\lambda=\rho(T)$.

Proof. Without loss of generality, assume $\rho(T)=1$. Just suppose 1 is not an eigenvalue of $T$. Define

$$
P_{t}=\left[\left(1-\sum t^{2} \begin{array}{c}
\overline{X_{i}} \\
\stackrel{\otimes}{X_{i}}
\end{array}\right)^{-1}\right]^{\psi} .
$$

Clearly, $P_{t}$ is well-defined, continuous, and finite for $t \in[0,1]$. Let $L_{t}=Q\left(P_{t}\right)$. For each $0 \leq t<1$, we see that $L_{t}-t^{2} \sum X_{i} L_{t} X_{i}^{*}=I$, by Proposition 4.1 because $\rho(t X)<1$. So, by continuity,

$$
L_{1}-\sum X_{i} L_{1} X_{i}^{*}=I>0 .
$$

Therefore, by Theorem 1.9, we see that $\hat{\rho}(X)=\rho(T)<1$, which is a contradiction.

To prove that the maximual spectrum actually contains a nonnegative element is somewhat more subtle. We now prove Theorem 1.2.

Proof. If $T$ is nilpotent, there is nothing to prove. Suppose $\rho(T)=1$. Recall $\Lambda$ is the closure of the subgroup of the torus $\mathbb{T}^{m_{T}}$ generated by the point $\tau=\left(\lambda_{1}, \ldots, \lambda_{m_{T}}\right)$ where the $\lambda_{i}$ are the elements of the maximal spectrum, where $m_{T}$ is the cardinality of the maximal spectrum of $T$. Recall there was a bijective map $T_{\lambda}$ from $\Lambda$ to the limit points of the sequence $\frac{T^{n}}{\left\|T^{n}\right\|}$. Note that $T_{\lambda}^{\psi} \geq 0$ by the Schur product property for the $\psi$ involution. Moreover, $T_{\lambda}^{\psi} \neq 0$. Now, recall,

$$
\hat{T}=\int_{\Lambda} T_{\lambda} \mathrm{d} \lambda=\lim _{N \rightarrow \infty} \frac{1}{N} \sum_{n=1}^{N} \frac{T^{n}}{\left\|T^{n}\right\|} .
$$

So, $\hat{T}^{\psi} \geq 0$ and $\hat{T}^{\psi} \neq 0$. By considering the Jordan decomposition of $T$, we see that any generalized eigenvector $v$ of $T$ corresponding to an eigenvalue which is not 1 must be sent the zero vector by $\hat{T}$ by looking that the formula for $\hat{T}$. Moreover, if 1 is an eigenvalue, but does not have maximal degeneracy index, $\hat{T}$ would also send any generalized eigenvector $v$ with eigenvalue 1 to 0 . Therefore, since $\hat{T} \neq 0,1$ must be an eigenvalue of $T$ with maximal degeneracy index. 
5.2. Structure of the $T_{\lambda}$. We now prove Theorem 1.3 .

Proposition 5.2. Let $T=\sum \overline{X_{i}} \otimes X_{i}$. Additionally, assume that $\hat{\rho}\left(X_{1}, \ldots, X_{d}\right)=1$.

(1) There is an $a \in \mathbb{N}$ such that for every $\lambda \in \Lambda$ there are $A_{\lambda, 1} \ldots A_{\lambda, a} \in$ $M_{n}(\mathbb{C})$ such that $T_{\lambda}=\sum_{j=1}^{a} \overline{A_{\lambda, j}} \otimes A_{\lambda, j}$.

(2) There are $B_{k} \in M_{n}(\mathbb{C})$ whose span is a nonzero ideal in the algebra generated by $X_{1}, \ldots, X_{d}$ such that $\hat{T}=\sum_{k} \overline{B_{k}} \otimes B_{k}$. Moreover, the $A_{\lambda, j}$ are contained in the span of the $B_{k}$ 's.

Proof. Note $T_{\lambda}^{\psi} \geq 0$. So

$$
T_{\lambda}^{\psi}=\sum \operatorname{vec} A_{\lambda, j}\left(\operatorname{vec} A_{\lambda, j}\right)^{*}
$$

So, since $\psi$ interchanges Kronecker products and outer products,

$$
T_{\lambda}=\sum_{j=1}^{a} \overline{A_{\lambda, j}} \otimes A_{\lambda, j} .
$$

Moreover, since

$$
\hat{T}^{\psi}=\int_{\Lambda} T_{\lambda}^{\psi} \mathrm{d} \lambda
$$

we see that $\hat{T}$ has the appropriate form and vec $A_{\lambda, j}$ is in its range for each choice of $\lambda$ and $j$. (That is, something is in the kernel of $\hat{T}^{\psi}$ if and only if it is in the kernel of every $T_{\lambda}^{\psi}$.) Now, it remains to be seen that the $B_{k}$ span an ideal. Recall that $\hat{T} T=T \hat{T}=\hat{T}$. That is,

$$
\sum_{i} \sum_{k} \overline{X_{i} B_{k}} \otimes X_{i} B_{k}=\sum_{i} \sum_{k} \overline{B_{k} X_{i}} \otimes B_{k} X_{i}=\sum_{k} \overline{B_{k}} \otimes B_{k} .
$$

By taking the $\psi$ map of the relation, we see that

$\sum_{i} \sum_{k} \operatorname{vec} X_{i} B_{k}\left(\operatorname{vec} X_{i} B_{k}\right)^{*}=\sum_{i} \sum_{k} \operatorname{vec} B_{k} X_{i}\left(\operatorname{vec} B_{k} X_{i}\right)^{*}=\sum_{k} \operatorname{vec} B_{k}\left(\operatorname{vec} B_{k}\right)^{*}$,

which implies that each $X_{i} B_{k}$ and $B_{k} X_{i}$ is in the span of the $B_{k}$ 's and therefore they span an ideal in the algebra generated by the $X_{i}$ 's.

We now need the following lemma.

Lemma 5.3. Let $U=\sum \overline{A_{k}} \otimes A_{k}, V=\sum \overline{B_{k}} \otimes B_{k}$. If the $B_{k}$ 's are contained in the span of the $A_{k}$ 's then there is an $\varepsilon$ such that $\phi_{U}(H) \geq$ $\varepsilon \phi_{V}(H)$.

Proof. Note there is an $\varepsilon$ such that

$$
U^{\psi} \geq \varepsilon V^{\psi}
$$


So $(U-\varepsilon V)^{\psi}$ is positive semi-definite. Therefore, $U-\varepsilon V=\overline{C_{k}} \otimes C_{k}$ and so $\phi_{U-\varepsilon V}$ is a completely positive map.

We now prove Theorem 1.4 .

Proposition 5.4. Let $T=\sum \overline{X_{i}} \otimes X_{i}$. Additionally, assume that $\hat{\rho}\left(X_{1}, \ldots, X_{d}\right)=1$. If $X_{1}, \ldots, X_{d}$ generate the full algebra of $n$ by $n$ matrices, then $\hat{T}$ has rank 1 . Moreover, in such a case, $\hat{T}=\sum_{k} \overline{B_{k}} \otimes B_{k}$ where the $B_{k}$ 's span $M_{n}(\mathbb{C})$. That is, the ideal described in Theorem 1.3 is equal to the whole algebra, as the only nonzero ideal in $M_{n}(\mathbb{C})$ is $M_{n}(\mathbb{C})$ itself.

Proof. First note that $\hat{T}=\sum_{k} \overline{B_{k}} \otimes B_{k}$ where the $B_{k}$ 's span $M_{n}(\mathbb{C})$ by Theorem 1.3 and the fact that $M_{n}(\mathbb{C})$ is simple. Note that is such a case, $\phi_{\hat{T}}(H) \geq \varepsilon(\operatorname{Tr} H) I$ by Lemma 5.3, since $(\operatorname{Tr} H) I=\sum E_{i j} H E_{i j}^{*}$. We will work to establish that there is a positive semidefinite matrix $H$ such that $\phi_{\hat{T}}(H)$ has a kernel. If $\hat{T}^{2}=0$, for any postive matrix $H$, either $H$ is in the kernel or $\phi_{\hat{T}}(H)$ is.

Now, suppose $\hat{T}^{2}=\hat{T}$. Just suppose the rank of $\hat{T}$ is greater than 1 . Now there must be $v_{1}, v_{2} \in \mathbb{C}^{n}$ such that $T \overline{v_{j}} \otimes v_{j} \neq 0$, and $T \overline{v_{1}} \otimes v_{1}$ and $T \overline{v_{2}} \otimes v_{2}$ are linearly indepenent. Let $W_{1}$ and $W_{2}$ be matrices such that vec $W_{j}=T \overline{v_{j}} \otimes v_{j}$. Note $W_{j}=\sum_{i} X_{i} v_{j}\left(X_{i} v_{j}\right)^{*}$. Note $\phi_{\hat{T}}\left(W_{j}\right)=$ $W_{j}$. Since $W_{1}$ and $W_{2}$ are linearly independent positive semidefinite matrices, there is $W_{3}$ in their span which is positive semidefinite and singular. Moreover, $\phi_{\hat{T}}\left(W_{3}\right)=W_{3}$ which is singular. This contradicts the fact that $\phi_{\hat{T}}(H) \geq \varepsilon(\operatorname{Tr} H) I$.

By considering the form of $\hat{T}=\operatorname{vec} V(\operatorname{vec} W)^{*}$ from the above theorem, and the fact that $\hat{T}^{\psi}=\bar{W} \otimes V$ must be positive, we see that both the $V, W$ must be positive and, therefore we can take square roots. An elementary calulation gives that the appropriate conjugation by $V^{1 / 2}$ and $W^{1 / 2}$ transform the corresponding completely positive map into either a unital or trace preserving map respectively.

Corollary 5.5. Let $T=\sum \overline{X_{i}} \otimes X_{i}$. Additionally, assume that $\hat{\rho}\left(X_{1}, \ldots, X_{d}\right)=$ 1 , and $X_{1}, \ldots, X_{d}$ generate the full algebra of $n$ by $n$ matrices. By Theorem 1.4 .

$$
\hat{T}=\operatorname{vec} V(\operatorname{vec} W)^{*} \text {. }
$$

Then,

$$
\dot{X}=\left(V^{-1 / 2} X_{1} V^{1 / 2}, \ldots, V^{-1 / 2} X_{d} V^{1 / 2}\right)
$$

is a row co-isometry and

$$
\grave{X}=\left(W^{1 / 2} X_{1} W^{-1 / 2}, \ldots, W^{1 / 2} X_{d} W^{-1 / 2}\right)
$$


is a column isometry. Moreover, the corresponding $\phi_{\bar{T}}$ is unital and $\phi_{\grave{T}}$ is trace preserving.

5.3. Spectral structure of $\sum X_{i}^{\otimes 2 k}$. Blondel and Nesterov 3 commented that the eigenvalues of the matrix $\sum X_{i}^{\otimes 2 k}$ appeared to be structured. In Parrilo and Jadbabaie [16], it was shown that one can project $\sum X_{i}^{\otimes 2 k}$ onto some special invariant subspace which corresponds to the "symmetric algebra of a vector space." We will now briefly explain these phenomena.

We will restrict our attention to matrices over the reals. Let $\left(X_{1}, \ldots, X_{d}\right) \in$ $M_{n}(\mathbb{R})$. Let $Y_{k}=\sum X_{i}^{\otimes k}$. For any permutation $\sigma:\{1, \ldots, k\} \rightarrow$ $\{1, \ldots, k\}$, we define $P_{\sigma}$ to be the map which takes $v_{1} \otimes v_{2} \otimes \ldots \otimes v_{k}$ to $v_{\sigma(1)} \otimes v_{\sigma(2)} \otimes \ldots \otimes v_{\sigma(k)}$. A direct calculation gives the following.

Remark 5.6. $P_{\sigma} Y_{k}=Y_{k} P_{\sigma}$. Thus, if $v$ is an eigenvector for $Y_{k}$ with eigenvalue $\lambda$, then so is $P_{\sigma} v$.

We also note that one can derive the structure of $P_{\sigma}$ (and thus the invariant subspaces of $Y_{k}$ ) using classical representation theory of the symmetric group, although this is rather involved [24].

Moreover, in test cases, we saw that the eigenvalue with maximum modulus of $Y_{2 k}$ was always real and positive, and, in nontrivial examples, occured with multiplicity 1 , which reflects the general situation described in Theorem 1.2. In such a case, the eigenvector corresponding to the maximum eigenvalue must satisfy $P_{\sigma} v= \pm v$. (In test cases, we found that $P_{\sigma} v=v$.) In general, the space $V=\left\{v \mid P_{\sigma} v=v \forall \sigma\right\}$ is an invariant subspace of $Y_{k}$. In fact, it follows from Parrilo and Jadbabaie[16, Theorem 4.2] that

$$
\rho\left(X_{1}, \ldots, X_{d}\right) \leq \rho\left(\left.Y_{2 k}\right|_{V}\right)^{1 / 2 k} \leq \rho\left(Y_{2 k}\right)^{1 / 2 k} .
$$

We now remark that one can rephrase the above in terms of a certain action on polynomials. We the naturals action $\tau$ of each $X_{i}$ on a ring of polynomials $\mathbb{R}\left[e_{1}, \ldots, e_{n}\right]$ given by $\tau_{X_{i}} \cdot p(e)=p\left(X_{i} e\right)$ (here $e$ is the column vector with entries $e_{i}$.) One can check that the action of $Y_{k}$ on $V$ is isomorphic to the action $\sum \tau_{X_{i}}$ on homogeneous polynomials of degree $k$ in $\mathbb{R}\left[e_{1}, \ldots, e_{n}\right]$, denoted $\mathbb{R}\left[e_{1}, \ldots, e_{n}\right]_{k}$. That is, we have the following theorem which follows immediately from the Parrilo and Jadbabaie inequality above and Corollary 1.6.

Theorem 5.7. Let $\left(X_{1}, \ldots, X_{d}\right) \in M_{n}(\mathbb{R})^{d}$. Let $\tau_{X_{i}}$ denote the natural action of $X_{i}$ on polynomials $\mathbb{R}\left[e_{1}, \ldots, e_{n}\right]$.

$$
\rho\left(X_{1}, \ldots, X_{d}\right)=\lim _{k \rightarrow \infty} \rho\left(\left.\sum \tau_{X_{i}}\right|_{\mathbb{R}\left[e_{1}, \ldots, e_{n}\right]_{2 k}}\right)^{1 / 2 k} .
$$


We note that the total dimension of of the homogenous polynomials of degree $k$ in $n$ variables is equal to $h_{n, k}=\left(\begin{array}{c}n+k-1 \\ n-1\end{array}\right) \approx k^{n-1} / n$ !, so we can realize $\tau_{k}$ as an $h_{n, k}$ by $h_{n, k}$ matrix. Theorem 5.7 is equivalent to the method in Parrilo and Jadbabaie [16] called $\rho_{S R, 2 d}$, and gives a natural interpretation of $Y_{k}$ restricted to $V$.

\section{REFERENCES}

[1] A.A. Ahmadi, R. Jungers, P.A. Parrilo, and M. Roozbehani. Joint Spectral Radius and Path-Complete Graph Lyapunov Functions. SIAM J. Control and Optimization, 52(1):687-717, 2014.

[2] Vincent D. Blondel. The birth of the joint spectral radius: An interview with gilbert strang. Linear Algebra and its Applications, 428(10):2261 - 2264, 2008. Special Issue on the Joint Spectral Radius: Theory, Methods and Applications.

[3] Vincent D. Blondel and Yurii Nesterov. Computationally efficient approximations of the joint spectral radius. SIAM Journal on Matrix Analysis and Applications, 27(1):256-272, 2005.

[4] Vincent D. Blondel and John N. Tsitsiklis. The Lyapunov exponent and joint spectral radius of pairs of matrices are hard-when not impossible-to compute and to approximate. Mathematics of Control, Signals and Systems, 10(1):3140, 1997.

[5] Vincent D. Blondel and John N. Tsitsiklis. The boundedness of all products of a pair of matrices is undecidable. Systems and Control Letters, 41:2:135-140, 2000 .

[6] M.-D. Choi. Positive linear maps on $C^{*}$-algebras. PhD thesis, University of Toronto, 1972.

[7] I. Daubechies and J. Lagarias. Two-scale difference equations. i. existence and global regularity of solutions. SIAM Journal on Mathematical Analysis, 22(5):1388-1410, 1991.

[8] David E. Evans and Raphael Hegh-Krohn. Spectral properties of positive maps on c*-algebras. Journal of the London Mathematical Society, s2-17(2):345-355, 1978.

[9] A.E. Frazho. Complements to models for noncommuting operators. J. Funct. Anal., 59(3):445 - 461, 1984.

[10] J.W. Helton, I. Klep, S. McCullough, and N. Slinglend. Noncommutative ball maps. J. Funct. Anal., 257:47-87, 2009.

[11] R.A. Horn and C.R. Johnson. Matrix Analysis. Cambridge University Press, Cambridge, 1985.

[12] K. Kraus. General state changes in quantum theory. Ann. Phys., 64:311-335, 1971.

[13] Matthew Lagro, Wei-Shih Yang, and Sheng Xiong. A perron-frobenius type of theorem for quantum operations. Journal of Statistical Physics, 169(1):38-62, Oct 2017.

[14] M. Michaek and Y. Shitov. Quantum version of wielandts inequality revisited. IEEE Transactions on Information Theory, pages 1-1, 2019.

[15] Alexander Mller-Hermes, Daniel Stilck Frana, and Michael M. Wolf. Entropy production of doubly stochastic quantum channels. Journal of Mathematical Physics, 57(2):022203, 2016. 
[16] P. A. Parrilo and A. Jadbabaie. Approximation of the joint spectral radius using sums of squares. Linear algebra and its Applications, 428(10):2385-2402, 2008.

[17] J.E. Pascoe. An elementary method to compute the algebra generated by some given matrices and its dimension. Linear Algebra and its Applications, 571:132 $-142,2019$.

[18] G. Popescu. Isometric dilations for infinite sequences of noncommuting operators. Trans. Amer. Math. Soc., 316:523-536, 1989.

[19] G. Popescu. Von Neumann inequality for $\left(B(\mathcal{H})^{n}\right)_{1}$. Math. Scand., 68:292-304, 1991.

[20] G. Popescu. Similarity problems in noncommutative polydomains. J. Funct. Anal., 267(11):4446-4498, 2014.

[21] Maxim Raginsky. Entropy production rates of bistochastic strictly contractive quantum channels on a matrix algebra. Journal of Physics A: Mathematical and General, 35(41):L585-L590, oct 2002.

[22] G. C. Rota and G. Strang. A note on the joint spectral radius. Indag. Math., 22:379-381, 1960.

[23] Gian-Carlo Rota. On models for linear operators. Communications on Pure and Applied Mathematics, 13(3):469-472, 1960.

[24] B. Sagan. The symmetric group. Representations, combinatorial algorithms, and symmetric functions, 2nd ed., Graduate Text in Mathematics 203. Springer-Verlag, 2001.

[25] M. Sanz, D. Perez-Garcia, M. M. Wolf, and J. I. Cirac. A quantum version of wielandt's inequality. IEEE Transactions on Information Theory, 56(9):46684673, Sep. 2010.

[26] Robert Schrader. Perron-Frobenius theory for positive maps on trace ideals. In Mathematical physics in mathematics and physics (Siena, 2000), volume 30 of Fields Inst. Commun., pages 361-378. Amer. Math. Soc., Providence, RI, 2001.

[27] Jianhong Xu and Mingqing Xiao. A characterization of the generalized spectral radius with Kronecker powers. Automatica, 47(7):1530 - 1533, 2011. 